\title{
Normality, Freedom, and Distress: Listening to the Menopausal Experiences of Indian Women of Haryana
}

\author{
Vanita Singh and M. Sivakami
}

\begin{abstract}
INTRODUCTION
Menopause is a biological phenomenon marked by permanent cessation of menstruation. Because it is experienced differently across cultures, its meanings and management are highly contested. There are two popular and contrasting perspectives of menopause: biomedical and feminist (Hyde et al. 2010). The biomedical model constructs menopause as a disease of estrogen deficiency, while some feminists view menopause as a natural process shaped by social forces. However, both models are criticized for their failures to engage the viewpoints of individual women who experience menopause (Murtagh and Hepworth 2003). Ferguson and Parry (1998) argue that the medicalization of menopause has sidelined the voices of women in the current discourse, resulting in incomplete understanding of women's experience. Anthropologists and other social scientists, as well as epidemiologists, have highlighted the differences in the symptom experience of women from different cultures and different socioeconomic backgrounds (Beyene 1986; Flint and Samil 1990; WHO 1996; Lock 1993; Avis et al. 2001; Lock and Kaufert 2001). Although the biological changes associated with menopause are universal, menopausal experiences, including the reporting of menopausal symptoms, are largely explained by concerns about aging and the change in social roles once women attain menopause (Obermeyer 2000; Obermeyer, Ghorayeb, and Reynolds 1999; Zeserson 2001). Thus, there is a need to understand the uniqueness in the menopausal experience of women within the broader social context.
\end{abstract}




\section{Contested Meanings of Menopause}

The field of endocrinology, which enabled the extraction of synthetic estrogen, was established in 1930, leading to an era of interest in menopause by medical practitioners and researchers (Coney 1993). Menopause became popularly understood as hormone deficiency disease leading to loss of femininity. The movement promoting hormone replacement therapy (HRT) in the 1960s to help women remain 'feminine forever' is highly criticized by feminists for its ageist and sexist agenda (Bell 1987; Coney 1993). Approaches to menopause within the medical community, however, have evolved from treating women and their bodies without reference to social context. Bell (1987) traces the history of medicalization of menopause in the United States and identifies three models-biological, psychological, and environmental-guiding medical practice. The biological model defines menopause as "a physiological process caused by cessation of ovarian function," emphasizing hormone deficiency and thus treatment with estrogen or HRT. The psychological model believes that women's personalities affect their symptom experience, so psychotherapy is the appropriate line of treatment. According to the environmental model, women's symptoms are the result of stresses and strains posed by changing social roles and responsibilities during midlife; it proposes that women change their lifestyle and habits to manage menopausal symptoms. All three models have identified the cause of distress during menopause as existing within women and thus advocate for medical intervention for every menopausal woman. Though the environmental model acknowledges the impact of external factors, the solution proposed is internal (Bell 1987). Feminists critique medical models for devaluing older woman (Bell 1987; Coney 1993), presenting menopause as a 'hormonal deficiency disease,' and representing the menopause experience as universal and definitive, all of which have led to the promotion of HRT as the "elixir of youth" (Bell 1987; Coney 1993; Klein 1992).

The use of HRT for managing menopausal symptoms became more contentious after the Women's Health Initiative (WHI) study that linked HRT use with increased risk of breast cancer, endometrial cancer, and cardiac morbidity (Hyde et al. 2010). The WHI study was one of the largest prospective studies assessing the long-term impact of HRT on women's health; however, it was stopped prematurely due to the alarming findings of increased risk of breast cancer and cardiac diseases among study participants (Hammond 2005). The media picked up the study findings, and physicians became highly cautious of using HRT for their patients (Lazar et al. 2007). However, the study soon was criticized in medical, epidemiological, and social science literature for its design, sample selection, and the results not being significant (Derry 2004; Hammond 2005; Wren 2009; Gurney et al. 2014). The feminist-medical debate on the use of HRT for managing menopause resurfaced with the failure of the WHI study. However, we believe strongly that listening to women's own voices is important to further our understanding of menopause. Hence, 
the focus of this current study is to bring out the uniqueness in the experiences of menopause among Indian women of Haryana and to place them within the broader social context.

The next section discusses the literature on menopause in India. We then explain the methodology adopted for this study, followed by a discussion of our findings and the three narratives that emerged. In the discussion section, we illuminate our findings using anthropological and sociological theories around life transitions and illness. We assert that the meanings of menopause are created by the complex interplay of social, cultural, and biological factors.

\section{Menopause in the Indian Context}

India, like many nations, is governed by patriarchal values where women's voices are often ignored or muted (Patel 2007; Syamala and Sivakami 2005). One area in which their experiences are silenced is the menstrual cycle. Menopause and menarche are taboos; they are rarely discussed in Indian society, reflected by the dearth of studies on menopause in the Indian context. Studies on menopause in India have mainly focused on the differences in age at menopause and factors affecting it (Kriplani and Banerjee 2005; Syamala and Sivakami 2005; Sharma et al. 2007; Dasgupta and Ray 2009; Singh and Sivakami 2014). These differences are attributed to rural/urban residence, lifestyle factors, age, environment, education status, occupation status, and reproductive history (Stanford et al. 1987; Brambilla and Mckinlay 1989; Hill 1996; Hidayet et al. 1999; Harlow and Signorello 2000; Kriplani and Banerjee 2005; Syamala and Sivakami 2005; Dasgupta and Ray 2009; Singh and Sivakami 2014; Agarwal et al. 2018). Some studies found women who report no symptoms at all, while others report many symptoms, including hot flashes, night sweats, anxiety, irritability, loss of vision, joint pains, vaginal dryness, numbness, and tingling (Singh and Arora 2005; Bernis and Reher 2007; Dasgupta and Ray 2009; Singh and Sivakami 2014). There are two plausible explanations for this disparity: first, according to Aaron, Muliyil, and Abraham (2002), women do not report menopausal symptoms, as they link them with aging rather than menopause; and second, talking about menarche and menopause is still taboo in Indian culture.

The extant studies on the perception of menopause in India indicate that menopause is seen as positive, since the end of menstrual bleeding removes the societal restrictions that come with the cultural view that menstruation is polluting (Singh and Arora 2005). The negative views about menstruation and menstruating women have appeared in the accounts of women from Haryana (Singh 2006). Singh's (2006) study in rural Haryana of practices during menstruation found that only $0.4 \%$ of the sample population was using sanitary napkins to manage menstrual bleeding because of the difficulty in accessing menstrual products. This created a source of monthly distress for these women. Moreover, studies on poor women from India (George 1994) 
have reported the difficulties women face in maintaining hygiene and managing menstrual bleeding in poor living conditions with limited space and no indoor toilets. Systematic review and meta-analysis of 138 studies on menstrual hygiene management among adolescent girls in India between 2000 and 2015 point out that adolescent girls experience menstruation as "shameful and uncomfortable" due to lack of water, sanitation, and hygiene facilities, insufficient puberty education, and poor hygienic practices to manage menstruation (van Eijk et al. 2016). The same study also showed the societal issues faced by Indian adolescent girls in the form of various restrictions, which adds to the negative construction of menstruation. These difficulties construct menstruation as a monthly distress for these women, who later consider postmenopause as a "freedom from monthly tension" (Singh and Sivakami 2014). Also, because menstruation prevents them from entering places that are considered sacred or pure, menopause is considered a type of liberation in Haryana (Singh and Arora 2005; Singh and Sivakami 2014; van Eijk et al. 2016).

It is important to understand the meanings of menopause as constructed by women themselves as an alternative to overreliance on the medical discourse. Some have argued that the reporting of menopausal symptoms as well as their management is based on the studies of white women (Avis et al. 2001), and our policies reflect that. The policies need to be guided by the "local biologies" (Lock and Kaufert 2001) to ensure effective management of menopause. This study, undertaken to amplify the voices of menopausal women from lower socioeconomic strata, was conducted in the North Indian state of Haryana. Haryana is known for its gender discrimination practices, which are reflected in the state's sex ratio-that is, the ratio of males to females in a population. Sex ratio is a powerful indicator of social health in any society (Patel 2007), and Haryana's is the lowest in India.

\section{Methods and Participants}

In 2012, we conducted in-depth qualitative interviews with 28 postmenopausal women from the lower socioeconomic strata in the state of Haryana. We recorded information related to women's socioeconomic status, educational and working status, parity, breastfeeding practices, lifestyle and eating habits, and menopause status, using a structured interview schedule as part of a larger study focusing on sociocultural differences in the age at menopause and symptom experience (see Singh and Sivakami 2014). A subset of women who identified themselves as postmenopausal and who agreed to share their menopause experience in detail were part of the qualitative narratives that form the sample participants for this study. We use pseudonyms throughout the paper to maintain privacy and confidentiality. The interviews lasted for 45-60 minutes, and the first author made a detailed summary of the interviews. The first author is familiar with the local language, and thus women discussed their experience using their own words, in their own space and time. 
The women interviewed were between 45 and 60 years old. They identified themselves as postmenopausal if their menstruation had stopped more than a year ago. The mean age at menopause was 47 years for urban women and 44 years for rural women. Of the 28 postmenopausal women, three women reported having had hysterectomies and thus had surgical menopause, while the rest had conventional menopause. In terms of education, 17 of the women were illiterate, 5 had completed education up to primary level, and only 6 women were educated up to senior secondary level. Four women were widowed and the rest were currently married. Almost all had children of marriageable age or were already married. Many of them were grandmothers. Six of them were currently working as unskilled labor and the rest did not work outside the home.

After establishing a rapport with the women, we asked them to discuss their menopausal experience, and the topics ranged from the changes in their menstrual cycle, changes in body, managing menopause, family and work life, social support, peer comparisons, and local beliefs about menopause and its management. Their experiences ranged from having had no symptoms to suffering from a multitude of symptoms. The gamut of experiences suggested the role of social context in the uniqueness of each woman's experience. The narrative accounts of each woman were crafted from the information obtained during the interviews. We conducted narrative analysis to obtain rich insights into women's experience of menopause and identified themes and subthemes to gauge the significance of sociocultural context in the experience of menopause; we then linked them to theoretical models (Ryan and Bernard 2003). Three distinct but co-occurring narratives, discussed below, emerged from the detailed accounts of North Indian women from lower socioeconomic strata. We also read the narrative accounts repeatedly to identify five key themes grounded in the data: bodily changes or complaints, support system, visit to health provider, local beliefs about menopause and its management, and attitude toward menopause. The themes were then compared and contrasted across all interviews using constant comparison method (Corbin and Strauss 1990). We drew on sociological and anthropological theories of illness and transition to discuss the differences and similarities across narratives.

Ultimately, the three distinct but co-occurring narratives that emerged were: menopause as a normal life transition, menopause as distress because it's taboo, and menopause as freedom from monthly distress and societal restrictions. We found that although these narratives co-occur as women reflect back or anticipate the future, one narrative dominated. We draw insights from anthropological and sociological theories of menopause, illness, and transition states (Kaufert 1982; Hyden 1997; Ballard, Kuh, and Wadsworth 2001) to illuminate the differences and similarities in narratives. We also compare and contrast the different narratives based on the five identified key themes. 


\section{FINDINGS}

\section{Menopause as a Normal Life Transition}

I do not have any issue . . . all of a sudden it stopped and I was happy. (Kamla, 49, rural resident)

Accounts of menopause as a normal life transition showed that women tend to normalize their symptoms by peer comparisons and by acknowledging the universality of menopause. Women used phrases like "every woman's issue" and "it's like childbirth" to normalize the experience.

Every woman has to go through this, women are made to suffer, it's like child-birth you know. (Meena, 56, rural resident)

It's over now. . . . I had to suffer from heavy bleeding for six months which every woman has to suffer. . . I have heard that menopause happens this way only. (Jyoti, 47 , urban resident)

Many women reported having had no symptoms and abrupt cessation of menstruation; for these women, menopause was a natural transition. They considered menopause symptom-free; they mentioned joint pains or headaches but associated those with aging rather than menopause (Singh and Sivakami 2014). Similar results have been reported by other Indian studies as well, indicating that Indian women report fewer symptoms, as they frequently link menopausal symptoms with symptoms of aging (Aaron, Muliyil, and Abraham 2002).

In contrast to Western studies, where women are influenced by biomedical discourse and visit health providers to make sense of their experience and confirm their menopausal status (see Hyde et al. 2010), our study depends on "vernacular health theories": using popular beliefs and perceptions in local communities to understand illness or a life transition (Goldstein 2000) and to make sense of their bodily changes. These women depend on the affirmation of their community, as Kamla (49, rural resident) offers: "I discussed with other women in my community and they told me, it's menopause (mabina-bandh) for you. . . . ." The conception of menopause as a normal life transition also comes from the accounts of women who expressed the insignificance of menopause in their lives once their family was complete. Many women stated that they did not need the menstrual cycle past age 40 , as menstruation is equated with reproduction, especially among illiterate women. As one woman said, "Why you need this? . . Once your family is complete, you don't need it" (Gyani, 58, rural resident).

Biomedical discourse also influenced women's perception of early menopause. Few who went to health providers mentioned their fear of getting 
uterine cancer. Most of the women acknowledged that they suffered from unmanageable, heavy, and painful bleeding during the menopause phase; however, they call it normal. They believe that if the 'bad blood' were to remain inside the body, they may develop cancer. This suggests the power of vernacular health theories in normalizing distress, which is crafted by the medical discourse of menopause (Goldstein 2000). Menopause became insignificant for those women who were busy managing the chaos of life, most notably poor women, whose struggle every day to ensure that their families get fed takes priority over reflection on their own health issues. Reena (58, urban resident), a domestic servant who did not recall her menopause experience, reflects this view: "I don't remember how it stopped . . . it's been 10 years now. . . . I think I had no complaints. . . . At that time I was busy doing my job [domestic servant]. I used to work in 10 houses and was busy whole day." Seema (53, rural resident) is another woman who failed to recall her menopause experience: "I never had time to think about menopause and all. . . . I don't even remember exactly when I stopped menstruating. . . . I was too busy managing my household chores and managing field work [agricultural fields]."

Beena (49, rural resident) has a daughter of marriageable age, which in their culture is as soon as they turn 18. Beena is more concerned about the marriage of her daughter than her menopausal status. The latter has no bearing on her identity, while the former shapes her identity as a mother. She said, "It's a woman's issue which she has to bear. . . . Now, it's over but I have lot of other tensions. . . I have to arrange for my daughter's wedding, she is 26 now. . . . It's getting late." Here, menopause, as a midlife transition is overshadowed by other events, and it becomes a routine and normal midlife transition, consistent with Ballard, Kuh, and Wadsworth (2001) finding that other life transitions, such as changing relationships with one's partner or children or changes in work life, often overshadow the experience of menopause.

Most women in our study reported that menopause was an insignificant life event that either passed without any symptoms or without any symptoms that they remember. Those who did have unwanted symptoms normalized them by ascribing them universality, as reflected through peer comparisons and phrases such as "every woman has to suffer this" and "women are made to suffer."

\section{Menopause as Distress}

In this second thematic narrative, distress emerged mainly from somatic changes (heavy and painful bleeding, sleepless nights, irritability, anxiety, mood swings, and frequent headaches), often exacerbated by negative life events and an inability to share their experience with anyone. Rajni (52, urban resident) recalls her struggle with painful and heavy menstruation during her 
perimenopause phase: "I suffered during menopause for 2 years. . . . It was heavy and painful bleeding which kept me awake . . . [I] used to feel very hot, couldn't sleep, had frequent headaches . . . was feeling depressed. . . . . During that time my husband died ... It was a tough time.”

In Rajni's account, there is no mention of depression or other body changes emerging from menopause. Rather, she reports that perimenopause became tough at the point where her husband died. That is, it was the death of her husband and her inability to share her distress with anyone that made perimenopause a difficult time for her. Sarla (51, rural resident), who is currently postmenopausal, recalls suffering and difficulties during perimenopause, particularly managing menstrual bleeding and maintaining hygiene in the presence of a male family member (her son). In her culture, it is unacceptable to discuss issues such as menarche and menopause with male members, as she mentions,

It's a suffering. . . I suffered heavy and painful bleeding for nine years. . . I used to bleed continuously for 15-20 days, was exhausted. . . . I had sleepless nights due to fear of spoiling the bed-sheet. . . I I used to be so tired . . . had no energy to stand and work ... wished I had someone to understand my situation and help with household chores. . . My daughters were married by that time and I can't discuss all this with my son. ... Whenever I used to lie down in bed, he used to ask me what has happened to you ... and I had no answer. ... Now I am free from all such tensions.

Sarla's wish for someone to understand her situation points to a lack of emotional and social support in her life. Though she mentions a number of symptoms-sleeplessness, exertion, dizziness-she does not intend to seek medical intervention. Rather, she expressed the need to be understood. In Sarla's account, the narratives of freedom and distress coexist. Initially, her difficulties managing menopause and its associated symptoms in the presence of male members in her house made her feel distressed about menopause; however, once her period stopped completely, she felt liberated from monthly tensions. In her culture, menstruation or menopause is a women's issue and should not be discussed with men.

We found that women who lacked a support system were more likely to feel the distress of menopause. For Sita (54, urban resident), the postmenopausal phase became very difficult because she had no one with whom to share her grief over the death of her husband. Her complaint about God highlights her helplessness and distress. She complains of frequent headaches and joint pains:

For two years, I suffered from heavy bleeding and painful menstruation. . . . It was difficult, you know very difficult . . . it was painful. . . . Now I am not menstruating, but I have all sorts of pain, headache I get every day, my vision has diminished, all my joints ache. . . . My husband died three months back 
[tears roll down her eyes] . . my daughter-in-law is not good ... my son is busy with his own life. . . [ [There is no one] whom to tell my problem . . . even God doesn't listen to my prayers.

Similarly, Geeta (59, rural resident) remembers her menopause experience and calls it a "suffering." She mentions her frequent fights with her husband and even her son. She expresses the need to be understood in the following excerpt:

During menopause, I suffered a lot. . . . I had very heavy bleeding . . . which was unmanageable. ... I tried many home-remedies . . . but nothing worked. . . . I was unable to sleep, fully exhausted . . . didn't wanted to talk to anyone, became anxious, used to get irritated over small things . . . had frequent fights with my husband . . . and even with my son. . . . They don't understand what a woman is going through . . . and you can't explain them. . . . It was a difficult time.

On the other side, Gita (52, rural resident) boasts about her daughter-in-law and mentions that she is enjoying this carefree life as a grandmother:

There was very heavy bleeding before I stopped menstruating . . . but that's normal . . . every woman has to go through this. . . My daughter-in-law is very nice . . . she managed all the household chores at that time . . she helped me a lot. ... Now she works and I look after her kids ... now I am free and enjoying life.

\section{Menopause as Freedom}

The third thematic narrative carried two meanings of freedom: freedom from the stressful management of menstruation and freedom from societal restrictions. Most women acknowledged that managing heavy and painful menstrual bleeding is difficult at an older age, thus they are happy when menstruation ends. The management is further complicated by the taboos attached to menstruation and menopause, which leads them to suffer in silence. Gita (52, rural resident) mentions, "Three to four years back it stopped. . . . It's good that it stops when you become old." Women expressed their difficulties in managing the pain and chaos of menstruation every month and thus embraced menopause as entry into a carefree phase of life. Women are happy in their new roles (mother-in-law or grandmother), which they would have found difficult with menstruation. As Gita adds, "It's good it stopped before I became grandmother as it's difficult to manage menstruation in old age."

For some women, the narrative of distress and freedom co-occurred. When they reflect back on their experience during perimenopause, they call it "suffering" but they ended with phrases like "I am free now," "I am enjoying now," and "it's over now." For these women, the narrative of freedom has emerged from the narrative of distress. For instance, Nirali, (53, urban 
resident) mentions, "two to three months I was bleeding heavily and then it stopped. . . . It was difficult to manage . . . it was annoying, now it's over."

For most of the rural and some urban women, ending menstruation liberated them from the societal restrictions on entering sacred places and participating in sacred rituals like wedding pheras. Rekha (48, rural resident) recalls that she was barred from entering wedding mandap of her daughter. She mentions, "Even during my daughter's wedding I was menstruating and thus could not perform many rituals." Seema (47, rural resident) earns a living by organizing kirtans (spiritual gatherings). In her community, it is not acceptable that she attends a kirtan while she is menstruating, as menstruation is considered to be polluting. She is happy that she has reached menopause, as now she can plan and organize spiritual gatherings any time: "I am happy that it stopped. . . . I run a mandli (group) for performing spiritual gatherings (kirtan). . . . It was so difficult to manage when I was menstruating. . . . I cannot plan a kirtan when I am menstruating. . . . Now, I am free."

All of these women are happy that they have attained menopause. They can freely go to temple, plan outings, and lead a carefree life. Women were more concerned about their new roles as grandmothers, and they felt liberated from monthly distress; by contrast, Western studies report that women are more concerned about their fertility status (Nosek, Kennedy, and Gudmundsdottir 2012). Some sociological and anthropological studies have reported that Indian women enjoy greater control over resources when they enter postmenopause and when they become mothers-in-law (Kaufert 1982; Inhorn 2006; Patel 2007). Also, Indian women enjoy higher social status in post-reproductive years due to freedom from the so-called 'polluting' menstruation and power dynamics (Vatuk 1998, 289-306; Aaron, Muliyil, and Abraham 2002).

\section{DiscusSION}

The narratives of normality, distress, and freedom emerged from the accounts of North Indian women from the lower socioeconomic strata. Each narrative surfaced from the complex interaction of biological changes, changes in family and work life, social status changes, and cultural beliefs. Though the narratives were distinct, they co-occurred in the accounts of many women.

The narratives of normality and freedom suggest the positive attitude of these women toward menopause. Our findings are reflected in earlier studies from India that examined perceptions of menopause (Aaron, Muliyil, and Abraham 2002; Singh and Arora 2005). The studies reported that Indian women embrace menopause, as they are free from the societal restrictions and the 'polluting' effects of menstruation. The narrative of normality was influenced by cultural perceptions about menopause, shaped either by their personal experience or that of their community members (Aaron, Muliyil, and 
Abraham 2002). It has been found that societies that value fertility, youth, and sexual attractiveness view menopause negatively (Kaufert 1982; Khademi and Cooke 2003; Hall et al. 2016), while societies in which menopause is considered to be socially liberating embrace it (Aaron, Muliyil, and Abraham 2002; Singh and Arora 2005; Syamala and Sivakami 2005). In these settings, postmenopausal women enjoy greater self-esteem (Hall et al. 2016).

Menopausal normality also emerged from the insignificance of menopause in the lives of many women who were busy managing other chaos of life. Ballard, Kuh, and Wadsworth (2001) report similar findings from their study of menopause experience in social context. The authors argue that social events compete with menopause for attention, and significant life events often overshadow the menopause experience. We found that life events such as the death of a husband, the birth of a grandson, or the marriage of a son or daughter overshadowed the menopause experience. These major life events that reshaped family structures rendered menopause insignificant.

The distress of menopause reported by studies from the West is different from the narrative of distress that has emerged in this study. For Western women, the distress primarily emerges from the anticipation of aging, losing fertility, and becoming less attractive (de Salis et al. 2018; Nosek, Kennedy, and Gudmundsdottir 2012); their accounts seem to be influenced more by the biomedical perspective (Nosek, Kennedy, and Gudmundsdottir 2012). Conversely, in our study, distress stemmed mainly from difficulty in managing heavy and painful bleeding, further exacerbated by taboos attached to menopause. The grief and distress expressed by one of the rural women in our study was palpable, as she described being barred from her daughter's wedding mandap because she was menstruating. The distress in the lives of poor women from Haryana seems to stem from the complex interaction of patriarchy, gender, and poverty.

The narrative of distress as well as freedom has its roots in the patriarchal structure of Indian society-broadly defined as the domination of women by men-as illuminated in the review of ethnographic works by Inhorn (2006). Inhorn asserts that patriarchy has a demoting effect on women's health through both the "micropatriarchy" in a doctor-patient relationship and the "macropatriarchy" in the family structure, in which men exert domination over females of the house. Patriarchy is also seen as women being discriminated against and/or abused by their husbands. There is also an age dimension, as older women exert control over younger women and girls in the household, sometimes tormenting them for being infertile or not doing household chores (Inhorn 2006).

The three thematic narratives are interlinked. Narratives of normality and freedom dominated, and for most women freedom emerged from the narrative of distress. The narrative of distress, rooted in patriarchal values and practices, co-occurred with normality and freedom for many women. Women cannot share their menopausal experiences with men in the house because 
a woman is considered polluting while menstruating. Even the accounts of normality reflect the workings of patriarchy in the sense that women are supposed to bear the pain and show themselves as culturally competent. The narrative of freedom departs from normative and oppressive power structures, however, in the way that it challenges the dominant negative image of menopause found in medical discourses that cast menopause as disease in need of treatment.

As these narratives have emerged from the accounts of postmenopausal women, we must note that the phase of menopause per se may be influential in shaping the meaning of menopause. We may expect different findings if premenopausal and perimenopausal women were also part of this narrative analysis. Furthermore, the findings are based on the accounts of women from low socioeconomic strata and a single state in India. Future research may look into women of more diverse backgrounds.

In conclusion, this study identified a spectrum of menopausal experiences of low-income Indian women, whose voices are rarely heard. When we listen to women's own stories, located in the social context, we can capture what menopause actually means to women. In this case, the result found a complex interplay of social, cultural, and biological factors. From here, we can develop strategies of support that enable healthy and empowered aging that meets women's diverse needs.

\section{REFERENCES}

Aaron, Rita, Jayaprakash Muliyil, and Sulochana Abraham. 2002. "Medico-Social Dimensions of Menopause: A Cross-Sectional Study from Rural South India." National Medical Journal of India 15 (1): 14-17.

Agarwal, Anil K., Nirmala Kiron, Rajesh Gupta, Aditi Sengar, and Preeti Gupta. 2018. "A Study of Assessment of Menopausal Symptoms and Coping Strategies among Middle Age Women of North Central India." International Journal of Community Medicine and Public Health 5 (10): 4470-77.

Avis, Nancy E., Rebecca Stellato, Sybil Crawford, Joyce Bromberger, Patricia Ganz, Virginia Cain, and Marjorie Kagawa-Singer. 2001. "Is There a Menopausal Syndrome? Menopausal Status and Symptoms across Racial/Ethnic Groups.” Social Science and Medicine 52 (3): 345-56.

Ballard, Karen D., Diana J. Kuh, and Michael E. J. Wadsworth. 2001. "The Role of the Menopause in Women's Experiences of the 'Change of Life'." Sociology of Health \& Illness 23 (4): 397-424.

Bell, Susan E. 1987. "Changing Ideas: The Medicalization of Menopause." Social Science \& Medicine 24 (6): 535-42.

Bernis, Cristina, and David Sven Reher. 2007. "Environmental Contexts of Menopause in Spain: Comparative Results from Recent Research.” Menopause 14 (4): 777-87.

Beyene, Yewoubdar. 1986. "Cultural Significance and Physiological Manifestations of Menopause a Biocultural Analysis." Culture, Medicine and Psychiatry 10 (1): 47-71. 
Brambilla, Donald J., and Sonja M. McKinlay. 1989. "A Prospective Study of Factors Affecting Age at Menopause." Journal of Clinical Epidemiology 42 (11): 1031-39.

Coney, Sandra. 1993. The Menopause Industry: A Guide to Medicine's 'discovery' of the Mid-Life Woman. Australia: Spinifex Press.

Corbin, Juliet M., and Anselm Strauss. 1990. "Grounded Theory Research: Procedures, Canons, and Evaluative Criteria.” Qualitative Sociology 13 (1): 3-21.

Dasgupta, Doyel, and Subha Ray. 2009. "Menopausal Problems among Rural and Urban Women from Eastern India." Journal of Social, Behavioral, and Health Sciences 3 (1): 2 .

Derry, Paula S. 2004. "Hormones, Menopause, and Heart Disease: Making Sense of the Women's Health Initiative." Women's Health Issues 14 (6): 212-19.

van Eijk, Anna Maria, M. Sivakami, Mamita Bora Thakkar, Ashley Bauman, Kayla F. Laserson, Susanne Coates, and Penelope A. Phillips-Howard. 2016. "Menstrual Hygiene Management among Adolescent Girls in India: A Systematic Review and Meta-Analysis.” BMJ Open 6 (3): e010290.

Ferguson, Susan J., and Carla Parry. 1998. "Rewriting Menopause: Challenging the Medical Paradigm to Reflect Menopausal Women's Experiences.” Frontiers: A Journal of Women Studies 19 (1): 20-41.

Flint, Marcha, and Ratna Suprapti Samil. 1990. "Cultural and Subcultural Meanings of the Menopause." Annals of the New York Academy of Sciences 592 (1): 134-47.

George, Annie. 1994. "It Happens to Us: Menstruation as Perceived by Poor Women in Bombay." In Listening to Women Talk about Their Health: Issues and Evidence from India, edited by Joel Gittelsohn, Margaret E. Bentley, Pertti J. Pelto, Moni Nag, Saroj Pachauri, Abigail D. Harrison, and Laura T. Landman, 168-83. New Delhi, India: Har-Anand Publications.

Goldstein, Diane E. 2000. “'When Ovaries Retire’: Contrasting Women's Experiences with Feminist and Medical Models of Menopause." Health 4 (3): 309-23.

Gurney, Elizabeth P., Margaret J. Nachtigall, Lila E. Nachtigall, and Frederick Naftolin. 2014. "The Women's Health Initiative Trial and Related Studies: 10 Years Later: A Clinician's View." The Journal of Steroid Biochemistry and Molecular Biology 142: 4-11.

Hall, Lisa, Lynn Clark Callister, Judith A. Berry, and Geraldine Matsumura. 2016. "Meanings of Menopause." Journal of Holistic Nursing 25 (2): 106-18.

Hammond, Charles B. 2005. "The Women's Health Initiative Study: Perspectives and Implications for Clinical Practice." Reviews in Endocrine \& Metabolic Disorders 6 (2): 93-99.

Harlow, Bernard L., and Lisa B. Signorello. 2000. "Factors Associated with Early Menopause." Maturitas 35 (1): 3-9.

Hidayet, N. M., S. A. Sharaf, S. R. Aref, T. A. Tawfik, and I. I. Moubarak. 1999. "Correlates of Age at Natural Menopause: A Community-Based Study in Alexandria." EMHJ-Eastern Mediterranean Health Journal 5 (2): 307-19.

Hill, Kenneth. 1996. “The Demography of Menopause.” Maturitas 23 (2): 113-27.

Hyde, Abbey, Jean Nee, Etaoine Howlett, Jonathan Drennan, and Michelle Butler. 2010. “Menopause Narratives: The Interplay of Women's Embodied Experiences with Biomedical Discourses." Qualitative Health Research 20 (6): 805-15.

Hydén, Lars-Christer. 1997. "Illness and Narrative." Sociology of Health and Illness 19 (1): 48-69. 
Inhorn, Marcia C. 2006. "Defining Women's Health: A Dozen Messages from More Than 150 Ethnographies." Medical Anthropology Quarterly 20 (3): 345-78.

Kaufert, Patricia A. 1982. "Myth and the Menopause." Sociology of Health \& Illness 4 (2): 141-66.

Khademi, S., and M. S. Cooke. 2003. "Comparing the Attitudes of Urban and Rural Iranian Women toward Menopause." Maturitas 46 (2): 113-21.

Klein, Renate. 1992. "The Unethics of Hormone Replacement Therapy*." Bioethics News 11 (3): 24-37.

Kriplani, Alka, and Kaberi Banerjee. 2005. "An Overview of Age of Onset of Menopause in Northern India." Maturitas 52 (3-4): 199-204.

Lazar, Felipe, Jr., Lucia Costa-Paiva, Sirlei S. Morais, Adriana Orcesi Pedro, and Aarão Mendes Pinto-Neto. 2007. "The Attitude of Gynecologists in São Paulo, Brazil 3 Years after the Women's Health Initiative Study.” Maturitas 56 (2): 129-41.

Lock, Margaret. 1993. "The Politics of Mid-Life and Menopause: Ideologies for the Second Sex in North America and Japan." Knowledge, Power, and Practice: The Anthropology of Medicine and Every Day Life: 330-36.

Lock, Margaret, and Patricia Kaufert. 2001. "Menopause, Local Biologies, and Cultures of Aging." American Journal of Human Biology 13 (4): 494-504.

Murtagh, Madeleine J., and Julie Hepworth. 2003. "Feminist Ethics and Menopause: Autonomy and Decision-Making in Primary Medical Care." Social Science \& Medicine 56 (8): 1643-52.

Nosek, Marcianna, Holly Powell Kennedy, and Maria Gudmundsdottir. 2012. "Distress during the Menopause Transition: A Rich Contextual Analysis of Midlife Women's Narratives." Sage Open 2 (3). https://doi.org/10.1177/2158244012455178.

Obermeyer, Carla Makhlouf. 2000. "Menopause across Cultures: A Review of the Evidence." Menopause 7 (3): 184-92.

Obermeyer, Carla Makhlouf, Françoise Ghorayeb, and Robert Reynolds. 1999. "Symptom Reporting Around the Menopause in Beirut, Lebanon." Maturitas 33 (3): 249-58.

Patel, Tulsi. 2007. "The Mindset Behind Eliminating the Female Fetus." In Sex-Selective Abortion in India: Gender, Society and New Reproductive Technologies, 135-74. Thousand Oaks, CA: Sage.

Ryan, Gery W., and H. Russell Bernard. 2003. "Techniques to Identify Themes." Field Methods 15 (1): 85.

de Salis, Isabel, Amanda Owen-Smith, Jenny L. Donovan, and Debbie A. Lawlor. 2018. "Experiencing Menopause in the UK: The Interrelated Narratives of Normality, Distress, and Transformation." Journal of Women and Aging 30 (6): $520-40$.

Sharma, Sudhaa, Vishal R. Tandon, and Annil Mahajan. 2007. "Menopausal Symptoms in Urban Women." Alcohol 4: 3-41.

Singh, Amarjeet. 2006. "Place of Menstruation in the Reproductive Lives of Women of Rural North India." Indian Journal of Community Medicine 31 (1): 10.

Singh, Amarjeet, and Arvinder Kaur Arora. 2005. "Profile of Menopausal Women in Rural North India." Climacteric 8 (2): 177-84.

Singh, Vanita, and M. Sivakami. 2014. "Menopause: Midlife Experiences of Low Socio-Economic Strata Women in Haryana.” Sociological Bulletin 63 (2): 263-86.

Stanford, Janet L., Patricia Hartge, Louise A. Brinton, Robert N. Hoover, and Ronald Brookmeyer. 1987. "Factors Influencing the Age at Natural Menopause." Journal of Chronic Diseases 40 (11): 995-1002. 
Syamala, T. S., and M. Sivakami. 2005. "Menopause: An Emerging Issue in India." Economic and Political Weekly 40: 4923-30.

Vatuk, Sylvia. 1998. “The Indian Woman in Later Life: Some Social and Cultural Considerations." In Women's Health in India: Risk and Vulnerability, edited by Monica Das Gupta, Lincoln C. Chen, and T. N. Krishnan, 289-306. New Delhi, India: Oxford India Paperbacks.

World Health Organization. 1996. "Research on the Menopause in the 1990s: Report of a WHO Scientific Group.” Geneva: Switzerland. World Health Organization. https://apps.who.int/iris/handle/10665/41841.

Wren, Barry G. 2009. "The Benefits of Oestrogen Following Menopause: Why Hormone Replacement Therapy Should Be Offered to Postmenopausal Women." Medical Journal of Australia 190 (6): 321-25.

Zeserson, Jan Morgan. 2001. "How Japanese Women Talk about Hot Flushes: Implications for Menopause Research." Medical Anthropology Quarterly 15 (2): 189-205.

Open Access This chapter is licensed under the terms of the Creative Commons Attribution 4.0 International License (http://creativecommons.org/licenses/ by $/ 4.0 /$ ), which permits use, sharing, adaptation, distribution and reproduction in any medium or format, as long as you give appropriate credit to the original author(s) and the source, provide a link to the Creative Commons license and indicate if changes were made.

The images or other third party material in this chapter are included in the chapter's Creative Commons license, unless indicated otherwise in a credit line to the material. If material is not included in the chapter's Creative Commons license and your intended use is not permitted by statutory regulation or exceeds the permitted use, you will need to obtain permission directly from the copyright holder.

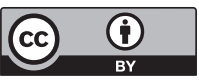

